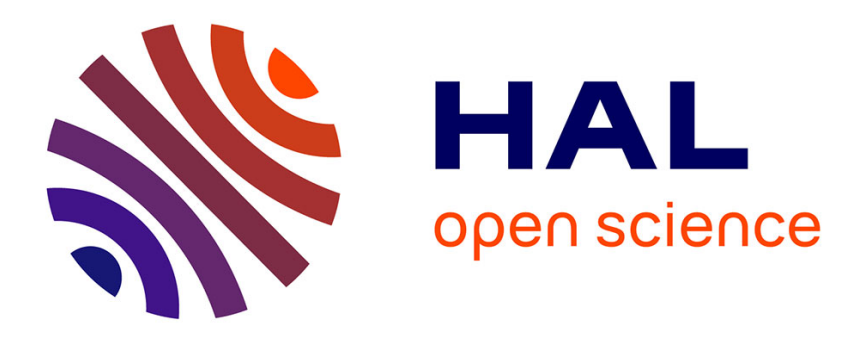

\title{
Rétrodiffusion Raman stimulée dans une fibre optique biréfringente
}

\author{
A. Saïssy, J. Botineau, L. Macon
}

\section{To cite this version:}

A. Saïssy, J. Botineau, L. Macon. Rétrodiffusion Raman stimulée dans une fibre optique biréfringente. Revue de Physique Appliquée, 1985, 20 (3), pp.129-136. 10.1051/rphysap:01985002003012900 . jpa00245314

\section{HAL Id: jpa-00245314 https://hal.science/jpa-00245314}

Submitted on 1 Jan 1985

HAL is a multi-disciplinary open access archive for the deposit and dissemination of scientific research documents, whether they are published or not. The documents may come from teaching and research institutions in France or abroad, or from public or private research centers.
L'archive ouverte pluridisciplinaire HAL, est destinée au dépôt et à la diffusion de documents scientifiques de niveau recherche, publiés ou non, émanant des établissements d'enseignement et de recherche français ou étrangers, des laboratoires publics ou privés. 
Classification

Physics Abstracts

$42.65 \mathrm{C}-42.80 \mathrm{M}$

\title{
Rétrodiffusion Raman stimulée dans une fibre optique biréfringente
}

\author{
A. Saïssy, J. Botineau et L. Macon \\ Laboratoire d'Electrooptique (*), Parc Valrose, 06034 Nice Cedex, France
}

(Reçu le 19 juillet 1984, révisé le 28 novembre, accepté le 30 novembre 1984)

\begin{abstract}
Résumé. - Nous étudions la rétrodiffusion Raman stimulée d'une impulsion lumineuse dans une fibre optique biréfringente. Le spectre de la lumière rétrodiffusée est composé de raies Stokes séparées de $450 \mathrm{~cm}^{-1} \mathrm{~d}^{\prime}$ une durée d'un ordre de grandeur inférieure à celle de l'impulsion incidente et une polarisation commandée par la puissance lumineuse excitatrice. Les principaux faits expérimentaux observés sont interprétés à l'aide d'un calcul de gain Raman prenant en compte la polarisation des différentes ondes en présence.
\end{abstract}

\begin{abstract}
We study the stimulated Raman backscattering of a quasi monochromatic light pulse travelling in a birefringent optical fiber. The backscattered spectrum contains several $450 \mathrm{~cm}^{-1}$ shifted Stokes lines with a temporal compression factor of ten and a polarization dependance with the excitation power. The main experimental features are explained with a Raman gain formalism taking into account the polarization of each wave.
\end{abstract}

\section{Introduction.}

La propagation de la lumière dans une fibre optique s'accompagne généralement de phénomènes de rétrodiffusion dont l'origine dépend aussi bien des caractéristiques de la fibre que de la lumière. Lorsque l'intensité lumineuse est importante, cette rétrodiffusion devient stimulée. Ainsi, une impulsion lumineuse intense de faible largeur spectrale interagit avec les phonons acoustiques du milieu pour créer de la rétrodiffusion Brillouin stimulée. Si l'interaction a lieu avec les phonons optiques on observe de la rétrodiffusion Raman stimulée (R.R.S.). Dans la présente note, nous étudions ce type de diffusion dans une fibre optique biréfringente. La R.R.S. a été étudiée il y a quelques années dans des fibres optiques non biréfringentes $[1,2]$; à notre connaissance, aucune étude de ce type n'a encore été réalisée sur des fibres biréfringentes. L'emploi d'une fibre biréfringente permet de mettre en œuvre un effet Kerr optique pour des puissances lumineuses excitatrices inférieures au seuil de la diffusion Raman stimulée, aussi est-il intéressant de connaître comment l'effet Kerr va modifier l'amplitude de la polarisation de la R.R.S.

Nous développons tout d'abord une théorie du gain Raman rétrodiffusé dans une fibre optique biréfringente. Nous présentons ensuite les caracté-

(*) Associé au CNRS no 190. ristiques expérimentales de la R.R.S. Nous étudions enfin comment interpréter ces résultats expérimentaux dans le cadre de la théorie précédente.

\section{Gain Raman en rétrodiffusion.}

Une onde lumineuse intense associée au champ électrique $\mathbf{E}\left(\omega_{\mathrm{p}}\right) \exp -j \omega_{\mathrm{p}} t$ se propageant dans un milieu ayant un mode de vibration Raman actif de pulsation $\omega_{\mathbf{R}}$ induit un gain positif pour une onde sonde $\mathbf{E}\left(\omega_{\mathrm{s}}\right) \exp -j \omega_{\mathrm{s}} t$ de pulsation $\omega_{\mathrm{s}}=\omega_{\mathrm{p}}-\omega_{\mathrm{R}}$. L'interaction des deux ondes ci-dessus peut être décrite par un terme de polarisation non linéaire $\mathbf{P}_{\mathrm{NL}}$ de composante spectrale

$$
\mathbf{P}_{\mathrm{NL}}\left(\omega_{\mathrm{s}}\right)=\chi^{(3)}\left(-\omega_{\mathrm{s}} ; \omega_{\mathrm{p}},-\omega_{\mathrm{p}}, \omega_{\mathrm{s}}\right) \mathbf{E}\left(\omega_{\mathrm{p}}\right) \mathbf{E} *\left(\omega_{\mathrm{p}}\right) \mathbf{E}\left(\omega_{\mathrm{s}}\right)
$$

$\tilde{\chi}^{(3)}$ étant une susceptibilité tensorielle d'ordre trois. La propagation de l'onde sonde est décrite par l'équation :

$$
\operatorname{rot} \operatorname{rot} \mathbf{E}-\left(\frac{\omega_{\mathrm{s}}}{c}\right)^{2} \varepsilon_{\mathrm{r}} \mathbf{E}=\mu_{0} \omega_{\mathrm{s}}^{2} \mathbf{P}_{\mathrm{NL}}
$$

$\varepsilon_{\mathrm{r}}$ étant la constante diélectrique relative du matériau, l'onde pompe (resp. sonde) se propageant dans la direction $\mathrm{O} z$ (resp.-Oz), $\mathrm{O} x$ et $\mathrm{O} y$ étant les axes de biréfringence de la fibre. Nous supposerons que les modes de la fibre sont transverses $\left(E_{z}=0\right)$, linéairement polarisés [3] et normalisés de manière à transporter chacun une puissance moyenne unité. Ecri- 
vons la décomposition modale des champs en présence :

$$
\begin{gathered}
\mathbf{E}\left(\omega_{\mathrm{p}}\right)=\sum_{v} A^{(v)}(z) \exp j \beta_{v}^{(\mathbf{p})} z \mathbf{E}_{\mathrm{tp}}^{(v)}(x, y) \\
\mathbf{E}\left(\omega_{\mathrm{s}}\right)=\sum_{v} B^{(v)}(z) \exp -j \beta_{v}^{(\mathbf{s})} z \mathbf{E}_{\mathrm{ts}}^{(v)}(x, y) \\
\iint \mathbf{E}_{\mathrm{t}}^{(\sigma)} \cdot \mathbf{E}_{\mathrm{t}}^{(v)^{*}} \mathrm{~d} x \mathrm{~d} y=\frac{2 \mu_{0} \omega}{\beta^{(v)}} \delta_{\sigma, v} .
\end{gathered}
$$

En négligeant la dérivée seconde de $B(z)$, l'équation de propagation de l'onde sonde devient :

$\frac{\mathrm{d}}{\mathrm{d} z} B^{(v)}(z)=-j \frac{\omega_{\mathrm{s}}}{4} \iint \mathbf{P}_{\mathrm{NL}}\left(\omega_{\mathrm{s}}\right) \cdot \mathbf{E}_{\mathrm{ts}} \mathrm{d} x \mathrm{~d} y \exp j \beta_{v}^{(\mathbf{s})} z$
Cherchons les solutions de cette équation lorsqu'à l'entrée de la fibre, l'onde pompe possède une polarisation rectiligne située dans le plan Oxy. La fibre étant constituée de silice amorphe, les termes non nuls du tenseur $\chi_{i j k l}$ sont ceux de la classe isotrope [4]. Les composantes de la polarisation non linéaire seront :

$P_{X}=\chi_{1111}(x x x)+\chi_{1122}(x y y)+\chi_{1212}(y x y)+\chi_{1221}(y y x)$
$P_{Y}=\chi_{1111}(y y y)+\chi_{1122}(y x x)+\chi_{1212}(x y x)+\chi_{1221}(x x y)$

avec :

$$
(i j k)=E_{i}\left(\omega_{\mathrm{p}}\right) E_{j}^{*}\left(\omega_{\mathrm{p}}\right) E_{k}\left(\omega_{\mathrm{s}}\right) .
$$

Lorsque les ondes pompe et sonde se propagent dans un seul mode dont le profil transverse $E_{\mathrm{tp}}$ ou $E_{\mathrm{ts}}$ est indépendant de la polarisation nous obtenons :

$$
\begin{aligned}
& \frac{\mathrm{d}}{\mathrm{d} z} B_{x}=-j \frac{\omega_{\mathrm{s}} R}{4}\left\{\left(\chi_{1111}\left|A_{X}\right|^{2}+\chi_{1221}\left|A_{Y}\right|^{2}\right) B_{x}+\chi_{1212} \mathrm{e}^{-j\left(\delta \beta(\mathrm{p})-\delta \beta^{(\mathrm{s})}\right) z} A_{y} A_{x}^{*} B_{y}\right\} \\
& \frac{\mathrm{d}}{\mathrm{d} z} B_{y}=-j \frac{\omega_{\mathrm{s}} R}{4}\left\{\left(\chi_{1111}\left|A_{y}\right|^{2}+\chi_{1221}\left|A_{x}\right|^{2}\right) B_{y}+\chi_{1212} \mathrm{e}^{j\left(\delta \beta(\mathrm{p})-\delta \beta^{(\mathrm{s})}\right) z} A_{x} A_{y}^{*} B_{x}\right\}
\end{aligned}
$$

où $\delta \beta^{(\mathbf{p}, \mathbf{s})}=\beta_{x}^{(\mathbf{p}, \mathbf{s})}-\beta_{y}^{(\mathbf{p}, \mathrm{s})}$ chiffre la biréfringence de la fibre pour les ondes $\mathrm{p}$ et $\mathrm{s}$,

$$
R=\iint\left|\mathbf{E}_{\mathrm{tp}}\right|^{2}\left|\mathbf{E}_{\mathrm{ts}}\right|^{2} \mathrm{~d} x \mathrm{~d} y
$$

chiffre le recouvrement entre les ondes $\mathrm{p}$ et $\mathrm{s}$. Les termes $(x y y)$ et $(y x x)$ n'ont pas été retenus car leur contribution proportionnelle à $\exp j\left(\delta \beta^{(\mathbf{p})}+\delta \beta^{(\mathrm{s})}\right) z$ possède un comportement oscillatoire important; par contre les termes $(y x y)$ et $(x y x)$ ont été conservés. La résolution du système ci-dessus nécessite la connaissance de l'évolution avec $z$ des composantes $A_{x}$ et $A_{y}$ de l'onde pompe. On posera par la suite $\delta \beta=\delta \beta^{(\mathrm{s})}-$ $\delta \beta^{(p)}$ pour chiffrer la différence de biréfringence do la fibre vis-à-vis des ondes $\mathrm{p}$ et $\mathrm{s}$.

2.1 COMPOSANTES DE L'ONDE POMPE CONSTANTES. Ceci se produit lorsque le gain Raman est faible, dans ce cas le transfert d'énergie de la pompe à la sonde est suffisamment petit pour que l'onde pompe puisse être considérée comme constante. $\mathrm{Si} \xi_{0} / 2$ et $\rho(0)$ désignent l'anglé de polarisation et l'amplitude de l'onde pompe à l'entrée de la fibre nous avons :

$$
A_{X}(z)=\rho(0) \cos \left(\xi_{0} / 2\right), \quad A_{Y}(z)=\rho(0) \sin \left(\xi_{0} / 2\right) .
$$

Afin de simplifier l'écriture, on écrira les coefficients de couplage intervenant dans le système différentiel sous la forme :

$$
\begin{aligned}
& {\left[\begin{array}{l}
\mu \\
\rho \\
\gamma
\end{array}\right]=\frac{\omega_{\mathrm{s}} R}{4} \rho^{2}(0)\left[\begin{array}{lll}
\chi_{1111} & \chi_{1221} & 0 \\
\chi_{1221} & \chi_{1111} & 0 \\
0 & 0 & 0,5 \chi_{1212}
\end{array}\right] \times } \\
& \times\left[\begin{array}{l}
\cos ^{2} \xi_{0} / 2 \\
\sin ^{2} \xi_{0} / 2 \\
\sin \xi_{0}
\end{array}\right] .
\end{aligned}
$$

Substituant aux 3 paramètres $(\mu, \rho-\delta \beta, \gamma)$ les paramètres $(U, P, Q)$ tels que :

$$
\begin{aligned}
& U=-0,5 j(\mu+\rho-\delta \beta) \\
& P=0,5 j(\mu-\rho+\delta \beta) \\
& Q=-0,5 j \sqrt{(\mu-\rho+\delta \beta)^{2}+4 \gamma^{2}}
\end{aligned}
$$

et à la variable $z$ la variable $t=z-L$ ( $L$ étant la longueur de la fibre), le système admet pour solution :

$$
\begin{aligned}
& B_{x}(t)=\mathrm{e}^{U t}\left[F^{(+)} \mathrm{e}^{Q t}+F^{(-)} \mathrm{e}^{-Q t}\right] \\
& B_{y}(t)=\mathrm{e}^{(U-j \delta \beta) t}\left[G^{(+)} \mathrm{e}^{Q t}+G^{(-)} \mathrm{e}^{-Q t}\right]
\end{aligned}
$$

avec :

$$
\begin{aligned}
& F^{(+)}=0,5\left[\left(1-\frac{P}{Q}\right) B_{x 0}-j \frac{\gamma}{Q} \mathrm{e}^{j \delta \beta L} B_{y 0}\right] \\
& F^{(-)}=0,5\left[\left(1+\frac{P}{Q}\right) B_{x 0}+j \frac{\gamma}{Q} \mathrm{e}^{j \delta \beta L} B_{y 0}\right] \\
& G^{(+)}=0,5\left[\left(1+\frac{P}{Q}\right) B_{y 0}-j \frac{\gamma}{Q} \mathrm{e}^{-j \delta \beta L} B_{x 0}\right] \\
& G^{(-)}=0,5\left[\left(1-\frac{P}{Q}\right) B_{y 0}+j \frac{\gamma}{Q} \mathrm{e}^{-j \delta \beta L} B_{x 0}\right]
\end{aligned}
$$

avec $\left(B_{x 0}, B_{y 0}\right)$ comme amplitude de l'onde sonde à l'extrémité $z=L$. L'évolution respective de $B_{x}(t)$ et $B_{y}(t)$ est commandée par les paramètres $P, Q$ et $\delta \beta$. Nous envisageons le cas particulier :

$$
P+Q \sim 0 \text { ou encore }|\gamma| \ll|\mu-\rho+\delta \beta| \text {. }
$$

La signification physique de cette condition est soit : $\chi_{1212} \ll \chi_{1111}$ (diffusion Raman faiblement dépolarisée), soit $\xi_{0} \sim 0$ ou $\pi$ (polarisation de l'onde 
pompe faiblement inclinée par rapport aux axes $O x$ et $\mathrm{Oy}$ ), ou encore $\delta \beta$ grand (fibre très biréfringente). Dans ce cas on a :

$B_{x}=B_{x 0} \exp -j \mu(z-L) \quad$ et $\quad B_{y}=B_{y 0} \exp -j \rho(z-L)$.

Lorsque l'écart de fréquence $\omega_{\mathrm{p}}-\omega_{\mathrm{s}}$ correspond à une fréquence de résonance Raman du milieu, les paramètres $\mu$ et $\rho$ sont imaginaires purs. Il apparaît donc un gain Raman en rétrodiffusion privilégiant l'axe sur lequel l'onde pompe est la plus intense.

Le mouvement électronique sous l'action de l'onde pompe se manifeste par une variation de la phase de l'onde sonde (effet Kerr optique) ainsi que de la phase de l'onde pompe (auto-effet Kerr) [6,7].

2.2 ONDE POMPE MODULÉE PAR AUTO-EFFET KERR. L'auto-effet Kerr est décrit par une polarisation non linéaire de composante spectrale,

$$
\begin{array}{r}
\mathbf{P}\left(\omega_{\mathbf{p}}\right)=\chi^{(\mathbf{A K})}\left(-\omega_{\mathrm{p}}, \omega_{\mathrm{p}},-\omega_{\mathrm{p}}, \omega_{\mathrm{p}}\right) \times \\
\times \mathbf{E}\left(\omega_{\mathrm{p}}\right) \mathbf{E}^{*}\left(\omega_{\mathrm{p}}\right) \mathbf{E}\left(\omega_{\mathrm{p}}\right) .
\end{array}
$$

La susceptibilité $\chi^{(\mathbf{A K})}$ est réelle et obéit aux relations :

$$
\chi_{1221}^{(\mathbf{A K})}=\chi_{1122}^{(\mathbf{A K})}=\chi_{1212}^{(\mathbf{A K})}=\frac{1}{3} \chi_{111}^{(\mathbf{A K})}
$$

Dans ce cas, l'amplitude de l'onde pompe dépend de $z$, soit :

$$
\begin{aligned}
& A_{x}(z)=\rho(0) \cos \left(\xi_{(z)} / 2\right) \mathrm{e}^{j \varphi_{x}^{(\mathrm{NL})}}, \\
& A_{y}(z)=\rho(0) \sin \left(\xi_{(z)} / 2\right) \mathrm{e}^{j \varphi_{y}^{(\mathrm{NL})}},
\end{aligned}
$$

où $\varphi^{(\mathrm{NL})}$ et $\xi / 2$ sont le déphasage non linéaire et l'angle de polarisation.

$\mathrm{La}$ résolution de l'équation de propagation pour l'onde pompe peut être conduite d'une manière identique à celle utilisée pour étudier la propagation de l'onde sonde en tenant compte du fait que le terme pompe n'est pas constant.

D'autre part, nous pouvons admettre que les modes propres de la fibre ne sont pas perturbés par la présence d'un effet Kerr optique, en effet il résulte des mesures antérieures [8] que la variation d'indice de réfraction induite par effet Kerr est négligeable devant la différence d'indice coeur-gaine de la fibre.

Introduisons les paramètres variables suivants :

$$
\eta=\varphi_{x}^{(\mathrm{NL})}-\varphi_{y}^{(\mathrm{NL})}+\delta \beta^{(\mathbf{p})} z
$$

(déphasage global entre les composantes de la pompe à l'abscisse $z$ ).

$$
R_{\mathrm{AK}}=\iint\left|\mathbf{E}_{\mathrm{tp}}\right|^{4} \mathrm{~d} x \mathrm{~d} y
$$

(intégrale de recouvrement de la pompe avec ellemême),

$$
S=\frac{\omega_{\mathrm{p}} R_{\mathrm{AK}}}{4 \delta \beta^{(\mathbf{p})}} \chi^{(\mathbf{A K})} \rho^{2}(0)
$$

On obtient un nouveau système différentiel décrivant l'évolution de la pompe.

$$
\begin{aligned}
& \frac{\mathrm{d} \eta}{\mathrm{d} Z}=1+2 S \cos \xi \sin ^{2} \eta \\
& \frac{\mathrm{d} \xi}{\mathrm{d} Z}=-S \sin \xi \sin 2 \eta
\end{aligned}
$$

avec $Z=\delta \beta^{(\mathbf{p})} z$.

Si on suppose que $\xi$ est sensiblement constant (cas d'une fibre fortement biréfringente), alors :

$$
\begin{aligned}
\eta & =\operatorname{Arctg}\left\{\left(1+2 S \cos \xi_{0}\right)^{-1 / 2} \operatorname{tg}\left[\left(1+2 S \cos \xi_{0}\right)^{1 / 2} \delta \beta^{(\mathbf{p})} z\right]\right\} \\
\operatorname{tg} \xi / 2 & =\operatorname{tg}\left(\xi_{0} / 2\right)\left\{1-\frac{2 S \cos \xi_{0}}{1+S 2 \cos \xi_{0}} \sin ^{2}\left[\left(1+2 S \cos \xi_{0}\right)^{1 / 2} \delta \beta^{(\mathbf{p})} z\right]\right\}^{1 / 2 \cos \xi_{0}}
\end{aligned}
$$

Dans le cas d'un coefficient $S$ petit, ces expressions deviennent :

$$
\begin{aligned}
\eta & =\operatorname{Arctg}\left\{\left(1-S \cos \xi_{0}\right) \operatorname{tg}\left[\left(1+S \cos \xi_{0}\right) \delta \beta^{(\mathbf{p})} z\right]\right\}, \\
\operatorname{tg} \xi / 2 & =\operatorname{tg} \xi_{0} / 2\left\{1-S \sin ^{2}\left[\left(1+S \cos \xi_{0}\right) \delta \beta^{(\mathbf{p})} z\right]\right\} .
\end{aligned}
$$

On voit que $\xi$ reste voisin de $\xi_{0}$, d'où la validité de l'expression de $\eta$. Revenons à l'étude de l'évolution de la sonde et considérons le cas où $\delta \beta$ est suffisamment grand pour que le couplage entre $B_{x}$ et $B_{y}$ soit négligeable. Dans ce cas :

$$
\begin{aligned}
& B_{X}(0)=B_{X}(L) \exp \left\{-j \frac{\omega_{\mathrm{s}} R}{4} \rho^{2}(0)\left[\chi_{1111} \int_{L}^{0} \cos ^{2} \xi / 2 \mathrm{~d} z+\chi_{1221} \int_{L}^{0} \sin ^{2} \xi / 2 \mathrm{~d} z\right]\right\} \\
& B_{Y}(0)=B_{Y}(L) \exp \left\{-j \frac{\omega_{\mathrm{s}} R}{4} \rho^{2}(0)\left[\chi_{1221} \int_{L}^{0} \cos ^{2} \xi / 2 \mathrm{~d} z+\chi_{1111} \int_{L}^{0} \sin ^{2} \xi / 2 \mathrm{~d} z\right]\right\}
\end{aligned}
$$


que l'on peut écrire :

$$
B_{X}(0)=B_{X}(L) \exp j(\mu L+\Delta \mu), \quad B_{Y}(0)=B_{Y}(L) \exp j(\rho L+\Delta \rho)
$$

avec :

$$
\Delta \mu=-\Delta \rho=\frac{\omega_{\mathrm{s}} R}{4} \rho^{2}(0)\left(\chi_{1111}-\chi_{1221}\right) \int_{0}^{\mathrm{L}}\left(\cos ^{2} \xi / 2-\cos ^{2} \xi_{0} / 2\right) \mathrm{d} z
$$

L'intégrale $\int_{0}^{L} \cos ^{2} \xi / 2 \mathrm{~d} z$ admettant pour développement limité :

$$
\frac{1}{\delta \beta^{(\mathbf{p})}} \cos ^{2} \xi_{0} / 2\left[1-\left(\cos \xi_{0}-\sin ^{2} \xi_{0} / 2\right) S\right] \operatorname{Arctg}\left\{\left(1-2 \sin ^{2} \xi_{0} / 2 S\right) \operatorname{tg}\left[\left(1+\cos \xi_{0} S\right) \delta \beta^{(\mathbf{p})} L\right]\right\} .
$$

Lorsque $S$ ou $\xi_{0}$ sont nuls, nous obtenons bien $\Delta \mu=0$ au premier ordre en $S$.

Pour l'onde sonde, une partie réelle dans la susceptibilité $\chi\left(-\omega_{\mathrm{s}}, \omega_{\mathrm{p}},-\omega_{\mathrm{p}}, \omega_{\mathrm{s}}\right)$ se traduit par la présence d'un effet Kerr [8], agissant sur l'indice de réfraction à la pulsation $\omega_{\mathrm{s}}$. Ceci peut être mis en évidence sur la polarisation de l'onde rétrodiffusée.

2.3 Polarisation DE L'ONDE RÉTROdIFFusée. - L'onde sonde rétrodiffusée a pour expression :

$$
\mathbf{E}^{(\mathbf{s})}\left(\omega_{\mathrm{s}}, t\right)=E_{\mathrm{t}}^{(\mathrm{s})}\left\{B_{\mathrm{OX}} \mathrm{e}^{-j\left(\tilde{\mu}+\beta_{x}^{(s)}\right) t} \hat{\mathbf{e}}_{x}+B_{\mathrm{OY}} \mathrm{e}^{-j\left(\tilde{\rho}+\beta_{y}^{(s)}\right) t} \hat{\mathbf{e}}_{y}\right\}
$$

où $\hat{\mathbf{e}}_{x}$ et $\hat{\mathbf{e}}_{y}^{\prime}$ sont les vecteurs unitaires portés par les axes de la fibre, $B_{\mathrm{OX}}$ et $B_{\mathrm{O} Y}$ les composantes de l'onde sonde à l'abscisse $L, \tilde{\mu}=\mu+\Delta \mu$ et $\tilde{\rho}=\rho+\Delta \rho$.

Soit $\hat{\mathbf{p}}=\cos \theta \hat{\mathbf{e}}_{x}+\sin \theta \hat{\mathbf{e}}_{y}$ la direction passante d'un polariseur placé sur le trajet de l'onde rétrodiffusée, l'amplitude de l'onde transmise par ce polariseur sera :

$$
\mathbf{E}^{(\mathbf{s})} \cdot \hat{\mathbf{p}}=E_{\mathrm{ts}}\left\{B_{\mathrm{Ox}} \mathrm{e}^{-j\left(\tilde{\mu}+\beta_{x}^{(s)) t}\right.} \cos \Theta+B_{\mathrm{Oy}} \mathrm{e}^{-j\left(\tilde{\rho}+\beta_{y}^{(s) t}\right.} \sin \Theta\right\} .
$$

Pour tenir compte de l'effet Kerr, nous prendrons $\tilde{\mu}$ et $\tilde{\rho}$ sous forme de nombre complexe $\tilde{\mu}=\tilde{\mu}^{\prime}-j \tilde{\mu}^{\prime \prime}$, de même pour $\tilde{\rho}$, dans ce cas :

$$
\left|\mathbf{E}^{(\mathbf{s})} \cdot \hat{\mathbf{p}}\right|^{2}=E_{\mathrm{ts}}^{2}\left\{\left|B_{O x}\right|^{2} \mathrm{e}^{-2 \tilde{\mu}^{\prime \prime} t} \cos ^{2} \theta+\left|B_{O y}\right|^{2} \mathrm{e}^{-2 \tilde{\rho}^{\prime} t} \sin ^{2} \theta+\mathrm{e}^{-\left(\tilde{\mu}^{\prime \prime}+\tilde{\rho}^{\prime \prime}\right) t}\left|B_{O x}\right|\left|B_{O y}\right| \sin 2 \theta \cos \Psi\right\}
$$

avec le déphasage total $\Psi$ donné par :

$$
\Psi=\left[-\left(\tilde{\mu}^{\prime}+\beta_{x}^{(s)}\right) t+\varphi_{0 x}\right]-\left[-\left(\tilde{\rho}^{\prime}+\beta_{y}^{(s)}\right) t+\varphi_{0 y}\right], \quad \varphi_{0 i}=\text { phase de } B_{0 i} .
$$

Cette intensité sera minimum pour un angle $\theta$ tel que :

$$
\operatorname{tg} 2 \theta=\frac{2\left|B_{\mathrm{O} x}\right|\left|B_{\mathrm{O} y}\right| \exp -\left(\tilde{\mu}^{\prime \prime}+\tilde{\rho}^{\prime \prime}\right) t}{\left|B_{\mathrm{O} x}\right|^{2} \mathrm{e}^{-2 \tilde{\mu}^{\prime \prime} t}-\left|B_{\mathrm{O} y}\right|^{2} \mathrm{e}^{-2 \tilde{\rho}^{\prime \prime} t}} \cos \Psi .
$$

La direction principale $\theta_{\mathbf{R}}$ de la vibration sera le complémentaire de l'angle $\theta$, soit :

$$
\operatorname{tg} \theta_{\mathrm{R}} \approx\left|\frac{B_{\mathrm{O} y}}{B_{\mathrm{Ox}}}\right| \exp -\left(\tilde{\rho}^{\prime \prime}-\tilde{\mu}^{\prime \prime}\right) t \cos \Psi \quad \text { pour } t \text { grand et } \tilde{\rho}^{\prime \prime} \ll \tilde{\mu}^{\prime \prime} .
$$

A l'entrée de la fibre $t=-L$, avec $\tilde{\mu}^{\prime \prime}$ et $\tilde{\rho}^{\prime \prime}$ positifs, le gain Raman de $B_{\mathrm{O} x}\left(\right.$ resp. $\left.B_{\mathrm{O} y}\right)$ sera $G_{x}=\tilde{\mu}^{\prime \prime} L$ (resp. $G_{y}=\tilde{\rho}^{\prime \prime} L$ ), d'où :

$$
\operatorname{tg} \Theta_{\mathrm{R}} \approx\left|\frac{B_{\mathrm{O} y}}{B_{\mathrm{Ox}}}\right| \exp \left(G_{y}-G_{x}\right) \cdot \cos \Psi .
$$

La polarisation de l'onde sonde rétrodiffusée sera déterminée principalement par l'anisotropie de gain $\delta G=G_{y}-G_{x}$ lorsque l'intensité de l'onde pompe est importante. En effet, $\operatorname{tg} \Theta_{R}$ dépend exponentiellement de $\delta G$, l'effet Kerr n'intervenant que pour moduler l'angle de polarisation autour de la valeur déduite de $\delta G$ et $\Psi=\delta \beta^{(s)} L$.

Un calcul ne prenant pas en compte l'existence de la partie réelle de la susceptibilité conduit directement à ce résultat, pour cela il faut que $\omega_{\mathrm{s}}-\omega_{\mathrm{p}}$ corresponde exactement à la fréquence de résonance Raman du milieu et que le gain soit important. 


\section{Montage expérimental}

Le dispositif expérimental est schématisé sur la figure 1. Il comporte un laser à colorant pompé par flash, la durée des impulsions est $1,25 \mu \mathrm{s}$ avec une puissance de $200 \mathrm{~W}$ et une fréquence de répétition de $10 \mathrm{~Hz}$. La longueur d'onde de travail a été choisie à $6000 \AA$ avec une polarisation horizontale. La fibre optique biréfringente de longueur $50 \mathrm{~m}$ est à coeur elliptique avec des axes égaux à $2 a=4,5 \mu \mathrm{m}$ et $2 b=6,5 \mu \mathrm{m}$. La lumière est couplée dans la fibre avec un objectif de microscope $(\times 20$ ou $\times 40)$, le rendement de couplage est de l'ordre de $50 \%$. En modifiant ce couplage, nous pouvons exciter les deux premiers modes de la fibre. L'extrémité de la fibre est montée dans un capillaire permettant d'orienter les axes de biréfringence de la fibre par rapport à la polarisation de la lumière incidente. La lumière rétrodiffusée est recueillie avec une lame de verre et analysée spectralement avec un réseau.

\section{Résultats et discussion.}

Le spectre de la lumière rétrodiffusée se compose, suivant la puissance lumineuse excitatrice, de trois ou quatre raies séparées de $450 \mathrm{~cm}^{-1}$, mode principal de diffusion Raman de la silice (Fig. 2). La durée des impulsions correspondant à la première raie Stokes est plus brève que celle de l'impulsion pompe $(1,25 \mu \mathrm{s})$. Elle est comprise entre 250 ns (Fig. 3) et $100 \mathrm{~ns}$, ce qui correspond à un facteur de compression compris entre 5 et 10. Pour nous assurer du rôle joué par la face de sortie de la fibre, nous avons fait varier le facteur de réflexion à l'interface fibre-air en plongeant l'extrémité de la fibre dans un liquide d'indice élevé $\left(\mathrm{CS}_{2}\right)$. Les différentes raies Stokes restent toujours en place, le processus de rétrodiffusion ne dépend donc pas d'une réflexion sur la face de sortie de la fibre. Dans le tableau I, nous avons reporté les longueurs de la fibre, les diamètres de cour et les durées d'impulsion correspondant aux références $[1,2]$. On constate que contrairement à ces références, nous sommes dans une situation où la longueur spatiale de nos impulsions est supérieure à celle de la fibre. L'augmentation du gain Raman lié à la conservation de la polarisation lumineuse par la fibre permet donc l'utilisation de fibres plus courtes, sans miroir de renvoi à la sortie et avec une puissance de seuil de l'ordre de $100 \mathrm{~W}$.

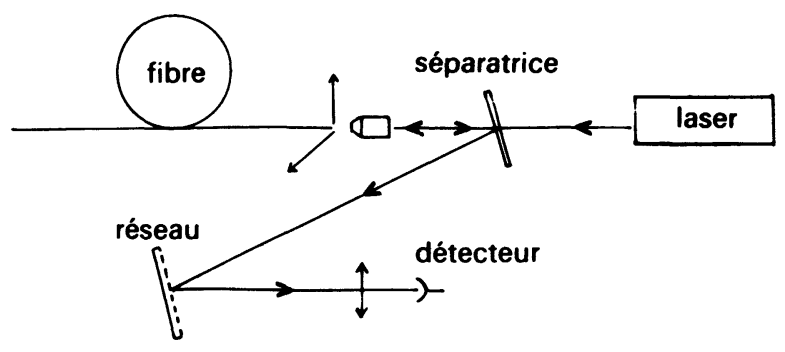

Fig. 1. - Dispositif expérimental.

[Experimental configuration.]

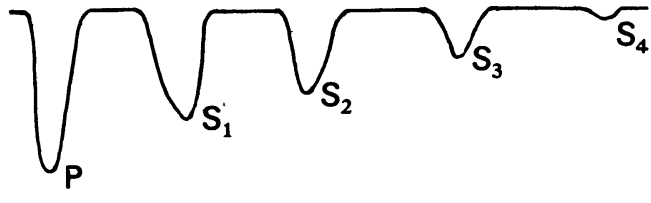

$100 \mathrm{~A}$

Fig. 2. - Spectre de la lumière rétrodiffusée. $P=$ onde pompe; $S_{i}=$ ondes Stokes.

[Backscattered spectrum. $P=$ pump wave; $S_{i}=$ Stokes waves.]

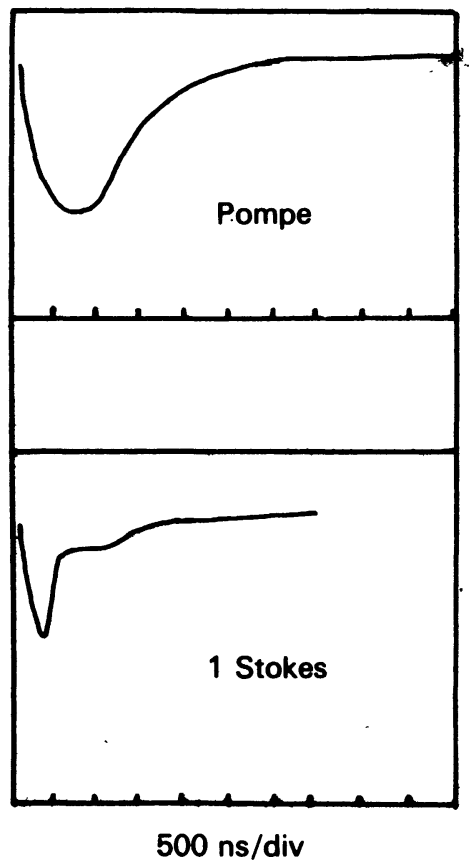

Fig. 3. - Profils temporels des ondes pompe et $1^{\mathrm{er}}$ Stokes.

[Temporal shape of pump and $1^{\text {rst }}$ Stokes waves.]

Le rendement énergétique de la conversion pompeStokes est de quelques pour cent; du fait de la compression d'impulsion, le rendement en puissance sera de quelques dizaines de pour cent. Pour avoir un gain Raman $G$ tel que $20<G<30$ il faut avoir une puissance pompe satisfaisant la relation :

$$
20<\frac{\gamma l_{\text {eff }} P}{A_{\text {eff }}}<30, \quad \text { où } \quad \gamma=\frac{2 \mu_{0}^{2} \omega_{\mathrm{s}}^{2} \omega_{\mathrm{L}}}{\beta_{\mathrm{s}} \beta_{\mathrm{L}}} \chi_{X X X X}^{(\mathbb{R})}
$$

est le coefficient de gain Raman égal à $10^{-11} \mathrm{~cm} / \mathrm{W}$ pour la silice [5]. La section effective de la fibre $A^{(e)}$ est égale à $\left(1,753 / U_{0}\right)^{2} a^{2}$ dans l'approximation gaussienne $\left(U_{0}=\right.$ paramètre de coeur de la fibre) soit $1,86 \times 10^{-7} \mathrm{~cm}^{2}$ avec $U_{0}=1,80$ et $a=2,5 \mu \mathrm{m}$. La longueur effective de la fibre est $l^{(\mathrm{e})}=\left(1-\mathrm{e}^{-\alpha L}\right) / \alpha$, 
Tableau I.

\begin{tabular}{|l|c|c|c|l|}
\hline & $\begin{array}{c}\text { Longueur de } \\
\text { fibre (m) }\end{array}$ & $\begin{array}{c}\text { Durée des } \\
\text { impulsions (ns) }\end{array}$ & $\begin{array}{c}\text { Diamètre de } \\
\text { coeur }(\mu \mathrm{m})\end{array}$ & Configuration \\
\hline Ref. [1] & 90 & 140 & 6 & Miroir en sortie \\
\hline Ref. [2] & 500 & 2000 & 80 & Simple passage \\
\hline & 50 & 1250 & 5 & $\begin{array}{l}\text { Simple passage } \\
\text { Biréfringence }\end{array}$ \\
\hline
\end{tabular}

soit $37,17 \mathrm{~m}$ pour $\alpha=54 \mathrm{~dB} / \mathrm{km}$ et $L=50 \mathrm{~m}$. Avec les chiffres ci-dessus nous obtenons $100 \mathrm{~W}<P<$ $150 \mathrm{~W}$, ce qui correspond expérimentalement à la gamme de puissance couplée dans la fibre.

Le calcul du gain Raman ci-dessus se situe dans un cas stationnaire, il ne rend donc pas compte de la compression d'impulsion, par contre il décrit les propriétés polarimétriques de la lumière rétrodiffusée. Lorsque l'onde pompe n'est plus polarisée suivant un axe de biréfringence, le coefficient de gain Raman manifeste son caractère anisotrope soit :

$$
\begin{aligned}
& \gamma_{X}(\xi / 2)=\gamma(0) \cos ^{2} \xi / 2+\gamma(\pi / 2) \sin ^{2} \xi / 2 ; \\
& \gamma_{Y}(\xi / 2)=\gamma(0) \sin ^{2} \xi / 2+\gamma(\pi / 2) \cos ^{2} \xi / 2 .
\end{aligned}
$$

L'étude polarimétrique du spectre de diffusion Raman spontanée de la fibre nous a montré que la bande $450 \mathrm{~cm}^{-1}$ est essentiellement polarisée [9], donc $\gamma(\pi / 2) \ll \gamma(0)$. Alors, pour un angle $\xi / 2$ différent de zéro, le gain Raman devient plus petit; expérimentalement ceci se traduit, pour une puissance pompe incidente donnée, par une plus grande sensibilité de la rétrodiffusion au couplage à l'entrée de la fibre.

Contrairement au spectre de diffusion codirective, le spectre de rétrodiffusion ne comporte pas de raie anti-Stokes. En effet, lorsque l'onde pompe est couplée dans le mode fondamental de la fibre avec une polarisation dirigée suivant l'axe lent on observe dans le spectre de diffusion codirective une onde anti-Stokes polarisée orthogonalement à l'onde pompe avec un écart de fréquence de l'ordre de $450 \mathrm{~cm}^{-1}$ par rapport à la fréquence pompe. Le mécanisme de création d'une onde anti-Stokes est du type mélange à quatre ondes [10], la relation d'accord de phase peut ici être satisfaite grâce à la biréfringence de la fibre, soit : $2 \beta_{X}^{(\mathrm{P})}=$ $\beta_{Y}^{(\mathbf{A S})}+\beta_{Y}^{(\mathbf{S})}$. La mesure du déplacement de fréquence $\Delta \sigma$ de l'onde anti-Stokes et la connaissance de la dispersion chromatique $D(\lambda)$ de la silice nous permet de connaître la biréfringence moyenne $\delta n$ de la fibre, la relation $2 \beta_{X}^{(\mathbf{P})}=\beta_{Y}^{(\mathbf{A S})}+\beta_{Y}^{(\mathbf{S})}$ nous donne $\delta n=$ $\lambda^{2} D(\lambda)(\Delta \sigma)^{2}$. Avec $\Delta \sigma \approx 450 \mathrm{~cm}^{-1}, D(\lambda)=0,060$, nous obtenons $\delta n \simeq 4,37 \times 10^{-5}$. Pour comparer le modèle théorique et l'expérience, il faut déterminer dans quelle situation nous nous trouvons. A partir de $\delta n$ on peut calculer $\delta \beta=\delta \beta^{(\mathrm{p})}-\delta \beta^{(\mathrm{s})}$ soit $\delta \beta=$
$2 \pi \delta n \Delta \sigma=0,12 \mathrm{~cm}^{-1}$ pour $\Delta \sigma=450 \mathrm{~cm}^{-1}$. D'autre part, $\mu^{\prime \prime}-\rho^{\prime \prime}$ est égal à $6 \times 10^{-5} \mathrm{~cm}^{-1}$ pour une amplification de $e^{30}$ sur $50 \mathrm{~m}$ de fibre, donc $\delta \beta \gg$ $\mu^{\prime \prime}-\rho^{\prime \prime}$. Donc la biréfringence est suffisamment importante pour que les composantes $B_{x}$ et $B_{y}$ de l'onde Stokes rétrodiffusée ne soient pas couplées. Pour comparer le modèle théorique à $\delta \beta$ grand avec l'expérience, nous avons étudié la polarisation de l'onde Stokes rétrodiffusée. Lorsque la polarisation de l'onde pompe n'est plus suivant un axe de biréfringence, nous relevons l'écart angulaire sur la position d'un polariseur correspondant à un minimum des intensités pompe réfléchie et onde Stokes rétrodiffusée. On constate que l'onde Stokes est encore fortement rectiligne, mais avec une polarisation dirigée suivant l'axe de biréfringence de la fibre la plus proche de la polarisation de l'onde pompe. Sur la figure 4 , nous avons porté l'écart des directions de polarisation des ondes pompe et Stokes en fonction de la direction de l'onde pompe. Les courbes théoriques sont déduites de l'expression $\operatorname{tg} \Theta_{\mathbf{R}}=\operatorname{tg}(\xi / 2) \exp \{-G \cos \xi\}$ pour $G=10,20$ et 30 . Dans la limite des erreurs expérimentales on peut admettre que les courbes théorique et expérimentale ont le même profil. Ceci confirme que c'est l'anisotropie de gain qui impose la polarisation de l'onde Stokes pour les petits angles $\xi / 2$; pour $\xi / 2$ voisin de $\pi / 4$ l'anisotropie de gain et l'effet Kerr optique s'annulent, il ne reste donc que la biréfringence naturelle de la fibre. L'effet Kerr optique redevient prépondérant lorsque l'on n'est plus en régime de diffusion stimulée. Pour l'onde pompe, l'influence de l'auto-effet Kerr est négligeable car la longueur de battement de la fibre $l_{\mathrm{b}}=\lambda \delta n / 2 \pi \sim 1,2 \mathrm{~cm}$ à $0,6 \mu \mathrm{m}$ est petite devant la longueur effective de la fibre $l^{(\mathrm{e})} \simeq 30 \mathrm{~m}$, avec un déphasage non linéaire très grand $\varphi^{\mathrm{NL}} \sim 10 \pi$ on obtient $S \simeq 4 \times 10^{-3}$, ce qui nous donne $\Delta \mu^{\prime \prime} \ll \mu^{\prime \prime}$. L'emploi d'une fibre très biréfringente permet donc d'éviter toute influence de l'autoeffet Kerr sur la rétrodiffusion Raman.

Pour une onde pompe polarisée rectilignement suivant l'axe lent de la fibre, on observe que le spectre de rétrodiffusion est plus intense lorsque le couplage à l'entrée de la fibre est réglé pour obtenir une raie anti-Stokes intense en sortie, en particulier la troisième raie Stokes rétrodiffusée augmente d'intensité. Cette 


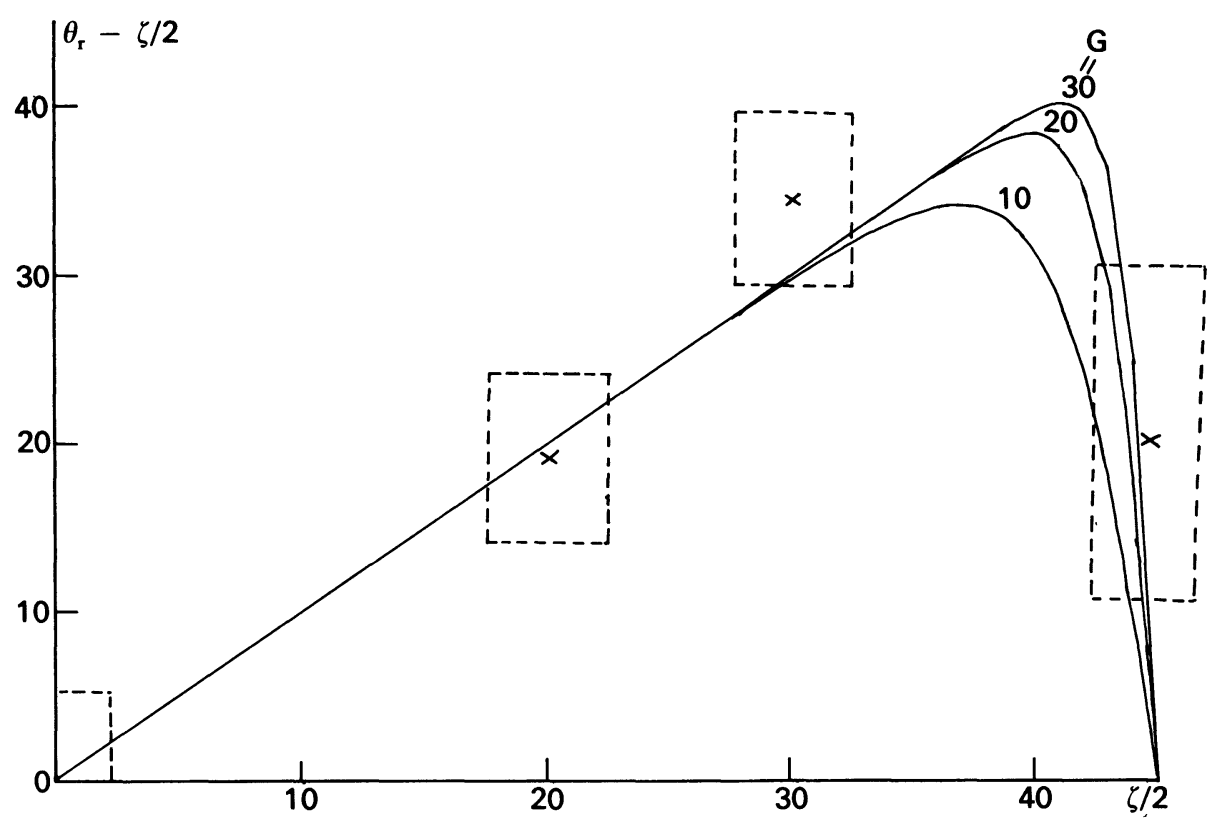

Fig. 4. - Ecart de polarisation des ondes pompe et Stokes en fonction de l'angle de polarisation de l'onde pompe : courbe théorique; $\times$ points expérimentaux; $G=$ gain Raman.

[Pump and Stokes waves polarization angle difference versus pump wave polarization angle : — theoretical curve ; × experimental points; $G=$ Raman gain.]

augmentation du gain à $\omega_{\mathrm{s} 3}=\omega_{\mathrm{p}}-3 \omega_{\mathrm{v}}$ résulte d'une amélioration du couplage à l'entrée et de la présence d'un processus de diffusion à quatre photons du type $\omega_{\mathrm{S} 3}=2 \omega_{\mathrm{S} 2}-\omega_{\mathrm{S} 1}$. En effet, la condition : $\beta_{\mathrm{S} 3}^{(2)}=\beta_{\mathrm{S} 2}^{(1)}+\beta_{\mathrm{S} 2}^{(2)}-\beta_{\mathrm{S} 1}^{(1)}$, où les indices (1) et (2) correspondent aux modes $\mathrm{LP}_{01}$ et $\mathrm{LP}_{11}$ est satisfaite pour une fibre de diamètre de cour $5,5 \mu \mathrm{m}$, de saut d'indice $\Delta \sim 3,67 \times 10^{-2}$ et des écarts de fréquence de $450 \mathrm{~cm}^{-1}$ pour $\omega_{\mathrm{s} 1}, \omega_{\mathrm{s} 2}$ et de $470 \mathrm{~cm}^{-1}$ pour $\omega_{\mathrm{s} 3}$. Ceci nous montre qu'un choix judicieux des paramètres opto-géométriques de la fibre permet d'augmenter l'intensité du spectre rétrodiffusé.

\section{Conclusion.}

Nous avons observé qu'une impulsion lumineuse intense $(100 \mathrm{~W})$ et suffisamment longue $(1,25 \mu \mathrm{s})$ crée une rétrodiffusion Raman stimulée à fort taux de compression [10] lors d'un simple passage dans une fibre optique biréfringente.

Nous avons calculé l'amplitude de la première onde Stokes rétrodiffusée et son comportement asymptotique suivant la biréfringence de la fibre. La mesure de la biréfringence de la fibre basée sur l'étude de la diffusion à quatre photons nous a donné $\delta n=4,37 \times 10^{-5}$, à cette biréfringence correspond une rétrodiffusion où les composantes de l'onde Stokes suivant les axes de la fibre sont découplées. Cette hypothèse a été vérifiée expérimentalement par l'étude polarimétrique de la lumière rétrodiffusée. A l'aide d'une méthode de perturbation, nous avons calculé l'influence de l'effet Kerr sur la rétrodiffusion Raman. Pour une fibre très biréfringente, cet effet n'agit pratiquement pas sur l'onde pompe, pour l'onde sonde en régime de diffusion stimulée, c'est l'anisotropie de gain qui impose la polarisation de l'onde Stokes. Cette dernière étant dirigée suivant l'axe le plus excité. Pour une configuration d'amplification où l'écart des fréquences des ondes pompe et sonde peut être décalé par rapport au maximum de la courbe de gain Raman, l'effet Kerr devrait intervenir plus fortement.

Une fibre optique biréfringente convenablement excitée, constitue donc un excellent générateur Raman, facilement convertible en amplificateur, fonctionnant en réflexion et capable de comprimer d'un ordre de grandeur des impulsions lumineuses en déplaçant leur fréquence et en modifiant leur polarisation. 


\section{Bibliographie}

[1] Chinlon Lin, Stolen, R. H., Appl. Phys. Lett. 29 (1976) 428.

[2] Lin, C. H., Marshall, B. R., Nelson, M. A., TheoBALD, J. K., Appl. Opt. 17 (1978) 2486.

[3] Gloge, D., Appl. Opt. 10 (1971) 2252.

[4] Flytzanis, C., Quantum Electronics, Ed. H. Rabin, C. L. Tang Vol 1, Part A (Academic Press).

[5] Stolen, R. H., IEEE J. Quantum Electron. 15 (1979) 1157.
[6] Stolen, R. H., Botineau, J., Ashkin, A., Opt. Lett. 7 (1983) 512.

[7] Saissy, A., Botineau, J., Ostrowsky, D. B., J. Optics 15 (1984).

[8] Saissy, A., Botineau, J., Ostrowsky, D. B., Appl. Opt. 22 (1983) 3869.

[9] Saissy, A., Botineau, J., Ostrowsky, D. B., J. Physique Lett. 44 (1983) 1035.

[10] Stolen, R. H., Bosch, M. A., Lin, C., Opt. Lett. G 213 (1981). 